\title{
REAL-TIME AND POST-PROCESSED GEOREFERENCING FOR HYPERPSPECTRAL DRONE REMOTE SENSING
}

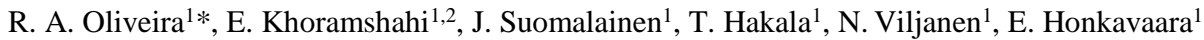 \\ ${ }^{1}$ Finnish Geospatial Research Institute, FGI, Finland - (raquelalvesdeoliveira, ehsan.khoramshahi, juha.suomalainen, teemu.hakala, \\ niko.viljanen, eija.honkavaara)@nls.fi \\ ${ }^{2}$ University of Helsinki, Department of computer science \\ ehsan.khoramshahi@helsinki.fi
}

Commission II, ICWG I/II

KEY WORDS: Photogrammetry, Real-time georeferencing, drone, Hyperspectral frame camera, Remote Sensing

\begin{abstract}
:
The use of drones and photogrammetric technologies are increasing rapidly in different applications. Currently, drone processing workflow is in most cases based on sequential image acquisition and post-processing, but there are great interests towards real-time solutions. Fast and reliable real-time drone data processing can benefit, for instance, environmental monitoring tasks in precision agriculture and in forest. Recent developments in miniaturized and low-cost inertial measurement systems and GNSS sensors, and Real-time kinematic (RTK) position data are offering new perspectives for the comprehensive remote sensing applications. The combination of these sensors and light-weight and low-cost multi- or hyperspectral frame sensors in drones provides the opportunity of creating near real-time or real-time remote sensing data of target object. We have developed a system with direct georeferencing onboard drone to be used combined with hyperspectral frame cameras in real-time remote sensing applications. The objective of this study is to evaluate the real-time georeferencing comparing with post-processing solutions. Experimental data sets were captured in agricultural and forested test sites using the system. The accuracy of onboard georeferencing data were better than $0.5 \mathrm{~m}$. The results showed that the real-time remote sensing is promising and feasible in both test sites.
\end{abstract}

\section{INTRODUCTION}

The use of drones (or Unmanned Aerial Vehicles - UAVs) and photogrammetric technologies have increased rapidly due to their potential in different application fields. In recent years, research has focused on real-time processing using drones, since many applications, such as natural disasters, search and rescue operations, modern precision agriculture, water quality and forest monitoring, can benefit from rapid and reliable information collection. Real-time photogrammetry has been discussed since decades ago (Haggren, 1986; Gruen, 1988 for example), but only in recent years, with the development of hardware and algorithms, real-time photogrammetry has become feasible. Drone real-time approaches can be related to an on-board data processing intended to an autonomous navigation and/or to data processing for user analyses.

Miniaturized and low-cost inertial measurement system (IMU) and GNSS sensors have entered to the market and being used in drone applications. Network based positioning, specifically with the Virtual Reference Station (VRS) concept, has been established in many countries, for example the Finnish network (Kirkko-Jaakkola et al., 2014). VRS technique enables few centimetre accuracy level in real-time without the high correlation between the distance of a rover and the closest reference station as in current single base real-time kinematic (RTK) systems (Rizos, 2003). In the literature, studies using GNSS based on RTK in drones have been carried out, for example, by Eling et al. (2015). Drones with RTK capability are also available in the market (SenseFly,2018; Skycatch, 2018)

Nowadays, visual sensors and algorithms have emerged as a low-cost, light-weight and low power consumption alternative for navigation and mapping. Since GNSS signal can be degraded under non-ideal environment conditions, new research and developments have increased in autonomous navigation. In the field of robotics, on-board motion estimation involves algorithms such as visual odometry (VO) and visual SLAM (Cadena et al., 2016). Bundle adjustment (BA) can be used to minimize the reprojection error of the camera orientations and $3 \mathrm{D}$ reconstruction. However, due to its high computational cost, BA has been performed incrementally for a set of keyframes (Kaess et al., 2012, Schneider et al., 2016). The accuracy and robustness of the estimations can be improved with information from inertial sensors and GNSS (Forster et al., 2017).

Real-time image processing can be performed using cloud computing (Luo et al., 2015; Kanga and Lee, 2016) or an onboard computer (Meier et al., 2011; Schneider et al., 2016). Generally, a cloud computer provides higher processing power than an on-board computer. However, data transfer which is an indispensable part of a cloud processing is a challenging factor since it depends on data transmission speed. The resolution and accuracy of the final products depend on the system processing power.

Although, miniaturized hyperspectral sensors have increased in the market (Honkavaara et al., 2013; Suomalainen et al., 2014; Oliveira et al., 2016), only few works have used multi/hyperspectral sensors in real-time remote sensing processing drone application (Koirala et al., 2017). Hyperspectral 2D-frame cameras can be collect registered, such as the Cubert UHD Firefly snapshot hyperspectral camera (Cubert, 2018) or unregistered bands, such as the Rikola Hyperspectral Camera, based on tuneable Fabry-Pérot Interferometer (FPI) (SENOP, 2018). The FPI camera acquires bands in time sequence mode; thus position and orientation have to be estimated for each band

\footnotetext{
* Corresponding author
} 
by direct georeferencing or/and image based band coregistration processes (Honkavaara et al., 2017)

Thus, investigating the potential and developing new systems and algorithms for the real-time drone remote sensing application is of great relevance and operational impact. In this study, real-time and post-processing data chains for image georeferencing have been implemented. The objective of this study is to evaluate the direct georeferencing solution. The results are compared with post-processing data, carried out based on pipelines developed by Honkavaara et al., 2013, 2018.

\section{METHODOLOGY}

\subsection{Platform and sensors}

Our designed multicopter is equipped with the FGI Aerial Image Reference System (FGI AIRS) consisting of an u-blox NEO-M8T GNSS receiver, a Vectornav VN-200 IMU, irradiance sensors, a Rasberry Pi 3 single-board computer and a 4G modem. The AIRS is connected to a camera system consisting of a hyperspectral 2D-format frame camera and an RGB camera. Hyperspectral 2D frame cameras allow an object to be imaged from different viewing angles, generating overlapping hyperspectral cubes. The sensor system has been designed to be operable using different drones with minimum of $2 \mathrm{~kg}$ payload capacity. For example, in Figure 1 we have a hexarotor drone with payload capacity of $4 \mathrm{~kg}$ and flight time of 20 minutes with $2 \mathrm{~kg}$ payload.

The processing design is presented in Figure 2 and is divided to the real-time and post-processing parts. The real-time processing provides approximate datasets with a few seconds delay after the capture of each image and the most accurate results are obtained by the post-processing. The main steps in the real-time component include: image pre-processing, GNSS/IMU trajectory calculation and irradiance data calculation; structure-from-motion (SFM) can be calculated to create the image orientations and sparse point cloud (section 2.3), and real-time orthorectification. Additionally, the band alignment is calculated when using Rikola camera. The realtime results can be send to the server or other application utilizing the data. The cloud processing can be performed on a dedicated server after transmitting data using the onboard modem. The post-processing includes the same steps as the realtime.

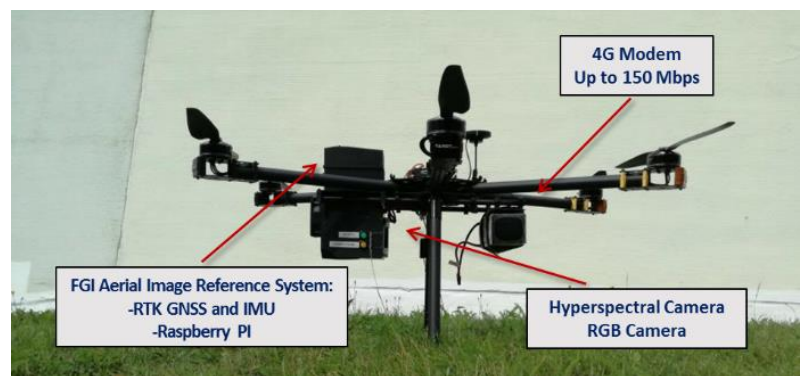

Figure 1. Implementation of the real-time hyperspectral drone system.

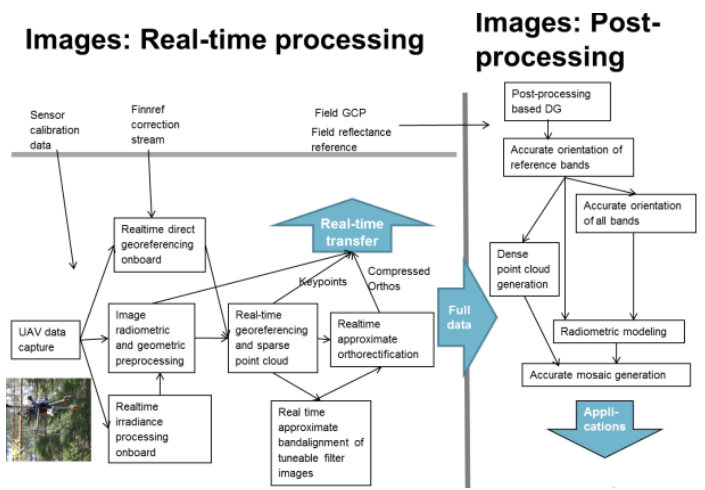

Figure 2. Real-time and post-processing chains for image based drone system.

\subsection{Real-time direct georeferencing}

An RTK georeferencing was established to allow real-time image georeferencing using the low cost GNSS/IMU system (ublox NEO-M8T; Vectornav VN-200 IMU). Differential RTK corrections from FinnREF are received by $4 \mathrm{G}$ modem to enable the onboard RTK processing.

The RGB and hyperspectral camera positions are synchronized to the GNSS/IMU-trajectory by recording the PPS signal from the GNSS unit and synchronization pulses for each exposure of each camera using the Raspberry Pi 3 GPIO. The PPS signal is used to calibrate the timestamps to GPS time at microsecond resolution. For the hyperspectral Rikola camera, a synchronization pulse is acquired for each band. This gives the platform positions and attitude (X, Y, Z, roll, pitch, and yaw) for each epoch, which are transformed to image positions using the boresight and lever arm information. The real time processing is using the open source software RTKLIB. The IMU outputs are Kalman filtered using the proprietary software.

\subsection{Incremental structure and motion estimation}

A real-time image-based solution for a multi-projective camera (MPC) system model has been developed to relatively estimate the georeferencing data. The proposed approach assumes that the drone's MPC is initially calibrated. FGI has a 3D calibration room equipped with coded targets to support the geometric calibration of MPCs. The details of the Photogrammetric calibration process of an MPC and specifications of FGI's calibration room could be seen in (Khoramshahi and Honkavaara, 2018 and Khoramshahi et. al 2017).

The real-time motion estimation approach is based on a sparse bundle block adjustment that employs SIFT tie points. The GPU of the board computer is responsible to compute the tie points features; here we employed the SiftGPU library (Wu, 2018). The resultant data is expressed in a local coordinate system that is based on the inner constraints that are a priori defined. This coordinate system is independent of the global coordinate system of GNSS and IMU. An inner-constraint adjustment is then employed to use the weighted observations of on-board GNSS and IMU (whenever such data is available).

A general incremental structure-from-motion based multisensor orientation system was developed. Sequential image matching by employing SIFT features computed on the GPU in a multi pyramid style is implemented to find a robust set of high-frequency tie points. Pairwise and multi-image local bundle block adjustment is implemented to improve the estimations of networks. Side overlaps have been calculated and 
taken into account to bolster the initial estimation of the local networks.

A sparse BBA is implemented by employing structure of Jacobian matrix. The main necessity of this implementation is to ensure enabling a fast solution that uses less Random Access Memory (RAM). Depending on the size of input images (10-20 MPix), the key point extraction and matching approximately requires 3-4 s on a regular laptop; due to some difficulties that occurs when the quality of textures and lightening is low, this processing requires up to $18 \mathrm{~s}$ to ensure a sufficient set of tie points by employing a patch-based matching approach. This matching is solely applied to the cases that the number of highquality matches is extremely low (less than 40 robust matches).

Approximate relative orientation is calculated in $<1 \mathrm{~s}$; these processing speeds can be further accelerated if necessary by techniques such as CPU-level parallelization. Outputs of the procedure are sparse point cloud representing the object approximate structure and exterior orientations of images in a local/global coordinate system. Incremental solution means that orientations of images and the object structure are formed image-by-image, giving a possibility to real-time applications The real-time solution is however approximate, the most accurate result is obtained by optimizing the solution with the full dataset. The method is capable for both options.

\subsection{Post-process workflow}

In the post-processing phase, the GNSS data is processed using the PPK mode of the RTKLIB and it processed relative to a ground station that is set up in the area of fight using single frequency GNSS receiver to collect data during the entire flight. The post-processed camera positions are introduced with the images in the commercial software Agisoft Photoscan Professional (AgiSoft LLC, St. Petersburg, Russia) for the final adjustment of the image position and orientation and for point cloud generation. An integrated geometric processing is used for the RGB images and hyperspectral images. The higher resolution RGB images are used to improve the accuracy of the geometric results. In the case of unregistered band camera, we selected reference bands in the photogrammetric software, since the orientation of each band of the FPI data cube (30-50 bands) would be computationally heavy. The rest of the bands are matched to the oriented bands using the band matching approach developed by Honkavaara et al. (2017). The camera exterior orientation parameters (EOPs) were estimated using the option "high" of the "Align" mode of PhotoScan (Align being the Bundle Adjustment). The camera calibration was performed with image orientation (self-calibration), using pre-calibrate values as initial approximation. Dense point clouds were generated using RGB images. Ground control points (GCPs) transform the data to the ETRS-TM35FIN coordinate system. The geometric accuracy was evaluated by using independent check points.

Radiometric modeling are required since the data capture is often executed in difficult conditions, for example, different illumination between cloudy and sunny, which impacts the spectral properties. In the radiometric processing of the hyperspectral data, the radiometric block adjustment method developed by Honkavaara et al. $(2013 ; 2018)$ used was. The reflectance transformation is carried out with empirical line method using reflectance panels in the area. The coordinate of the center of the panels are inputs in the block radiometric adjustment software and the observation of the $\mathrm{DN}$ values for each band and multiple views are integrated in the adjustment process. The panel coordinates can be estimated in the photogrammetric software as a tie point or using GNSS positioning survey. The final products are reflectance orthomosaics for each band. More details of the approach are given by Honkavaara et al., $(2013,2018)$.

\subsection{Test site and data sets}

Experimental testing of the georeferencing solution with the designed system was carried out in a grass test site located in Jokioinen $\left(68.814309^{\circ} \mathrm{N}, 23.505120^{\circ} \mathrm{E}\right)$, on 5 September 2017 , and in a forest test site located in Hyytiälä $\left(61.843724^{\circ} \mathrm{N}\right.$, $24.313492^{\circ} \mathrm{E}$ ), in 19 October 2017. The flying altitude in Jokionen was $50 \mathrm{~m}$ giving the GSD of $5 \mathrm{~cm}$ for the hyperspectral data and less than $1 \mathrm{~cm}$ for the RGB data. For forest test site, the flying altitude was $100 \mathrm{~m}$ and GSDs were 10 $\mathrm{cm}$ and $2 \mathrm{~cm}$ for hyperspectral and RGB data, respectively. The flight speed was $2.3 \mathrm{~m} / \mathrm{s}$ and $2.4 \mathrm{~m} / \mathrm{s}$ and flight time and 24 and 21 minutes. GCPs were measured in each area using Trimble $\mathrm{R} 10$ receiver.

The FPI camera prototype $2012 \mathrm{~b}$ was used to capture hyperspectral images on 36 bands in the range of 500-900 nm (Honkavaara et al., 2013). The camera has a CMOSIS CMV4000 RGB image sensor, focal length of $10.9 \mathrm{~mm}$ and $\mathrm{f}-$ number of 2.8. The time difference between adjacent exposures is $0.075 \mathrm{~s}$. The image size is 1024 x 648 pixels with a pixel size of $11 \mu \mathrm{m}$. The field of view (FOV) is $\pm 18^{\circ}$ in the flight direction, $\pm 27^{\circ}$ in the cross-flight direction, and $\pm 31^{\circ}$ at the format corner. The camera system weighs less than $700 \mathrm{~g}$. Up to now, the FPI hyperspectral camera does not support real-time accessing to the images, but it is suitable for our first analysis offline.

Another agricultural test site in Vihti, Hovi $\left(60^{\circ} 25^{\prime} 21^{\prime \prime} \mathrm{N}\right.$, $\left.24^{\circ} 22^{\prime} 28^{\prime \prime} \mathrm{E}\right)$ was used for the real-time image based georeferencing experiments. The flight was carried on 4 July 2017 with flying altitude of $140 \mathrm{~m}$. Only RGB images (GSD of $5 \mathrm{~cm}$ ) were used.

\section{EXPERIMENT AND RESULTS}

\subsection{Real time and post processed georeferencing}

Empirical testing of the georeferencing solution were performed for the two datasets. RTKLIB solutions can be processed using real-time (Real-time Kinematics - RTK) or post-processed process (Post-Processed Kinematics - PPK). Thus, the data were post processed to assess the camera positions obtained in realtime mode, and both RTK and PPK solutions were compared with the position estimated by the SfM commercial software.

Figure 3 shows the flight geometry of each study area and the location of GCPs. In Jokioinen testsite, the RTK solution obtained $21.2 \%$ of the points with ambiguity-fixed, while PPK had $92.2 \%$ of ambiguity solved. The root mean square error (RMSE) of the difference between RTK and PPK solution was $0.21 \mathrm{~m}$, in $\mathrm{N}, 0.18 \mathrm{~m}$, in $\mathrm{E}$, and $0.14 \mathrm{~m}$, in height.

In Hyytiälä forest test site, $46.5 \%$ of the camera locations were estimated with ambiguity-fixed RTK solution (Figure 3) and PPK had $71.5 \%$ of ambiguity solved. RTK and PPK solutions had RMSEs of the differences equal to $0.15 \mathrm{~m}$, in N, $0.26 \mathrm{~m}$, in $\mathrm{E}$, and $0.08 \mathrm{~m}$, in height. The ground station had not contributed for a better solution of PPK and it was not used in this case. The main reason was the influence of forest environment in the 
GNSS signal, since the ground station was located in a road surround by trees.

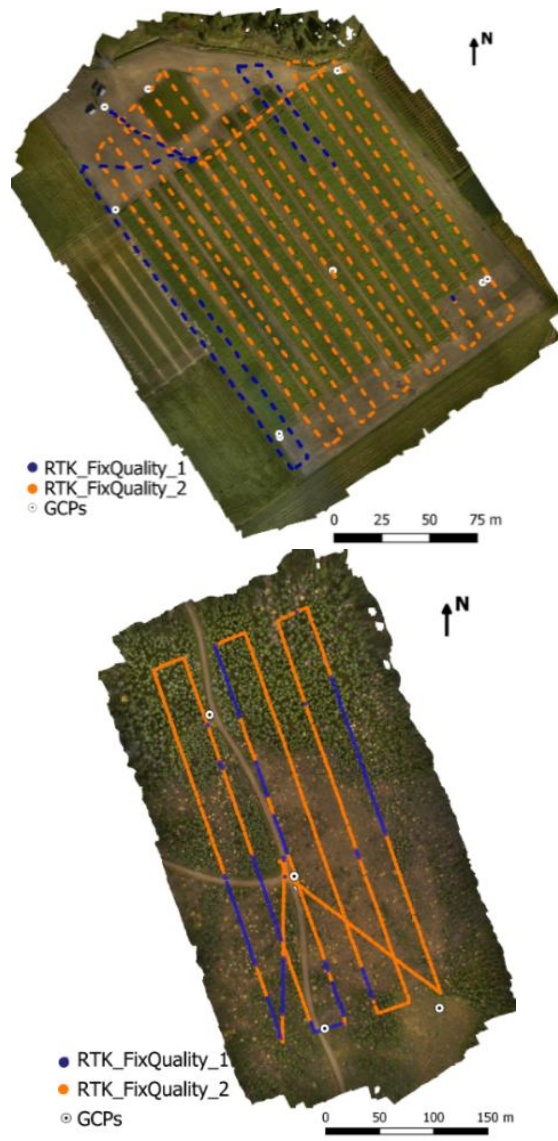

Figure 3. Test sites Jokioinen (top) and Hyytiälä (bottom) and ground control points. The background image are orthomosaics

generated from the RGB images collected by the designed system.

Table 1 presents RMSEs of the control points and check points obtained used in PhotoScan adjustment for each dataset. It is verified that the values were smaller than 1 GSD.

Figure 4 (top) shows the coordinate differences between the real time process and estimated camera positions in PhotoScan process, the RMSEs were $0.26 \mathrm{~m}$, in $\mathrm{E}, 0.22 \mathrm{~m}$, in $\mathrm{N}$, and 0.18 $\mathrm{m}$, in height, for Jokioinen data set. Hyytiälä assessment had RMSEs of $0.20 \mathrm{~m}$, in $\mathrm{E}, 0.31 \mathrm{~m}$, in $\mathrm{N}$, and $0.23 \mathrm{~m}$, in height (Figure 4 bottom). The RMSE for omega and pitch were approximated $1.6^{\circ}$ for both Jokionen and Hyytiälä flights, this value is within the expected quality of the sensor used. The estimation of the angle kappa is more complex and presented reduced quality. Figure 5 shows kappa estimated in PhotoScan and by the inertial sensor. During Jokioinen flight, the difference was within $\pm 10^{\circ}$, whereas in Hyytiälä the difference started around $30^{\circ}$ and it was reducing during the flight, reaching $3^{\circ}$ of difference (after 14 minutes of flight).

\begin{tabular}{|l|c|c|}
\hline & Jokioinen & Hyytiälä \\
\hline & GCP(10) & GCP(4) \\
$\mathrm{E}(\mathrm{m})$ & 0.012 & 0.003 \\
$\mathrm{~N}(\mathrm{~m})$ & 0.007 & 0.002 \\
$\mathrm{~h}(\mathrm{~m})$ & 0.015 & 0.005 \\
image(px) & 0.457 & 0.218 \\
\hline
\end{tabular}

Table 1. Accuracy (RMSE) of ground control point positions from results of post-processing bundle adjustment using Agisoft PhotoScan.

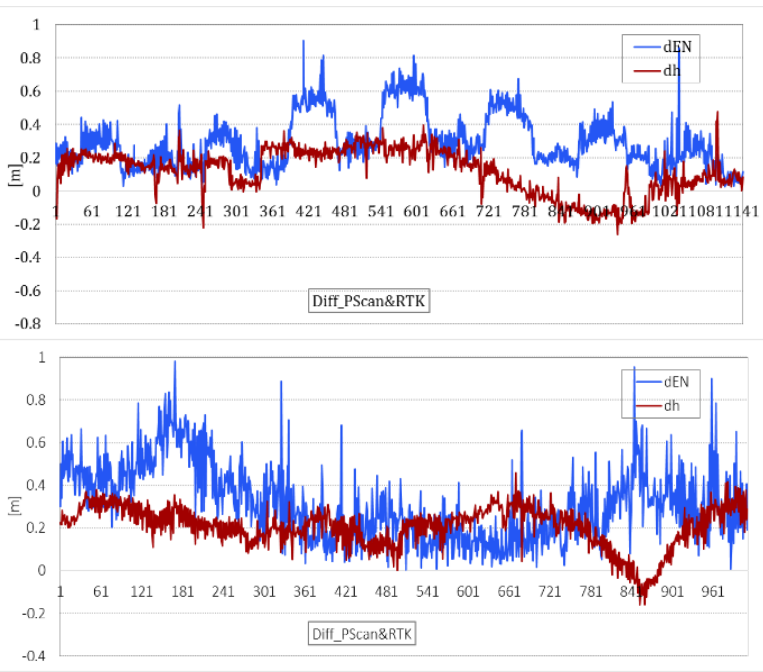

Figure 4. Differences of camera coordinates estimated in realtime processing (RTK) and in PhotoScan for Jokioinen (top) and Hyytiälä (bottom) area.

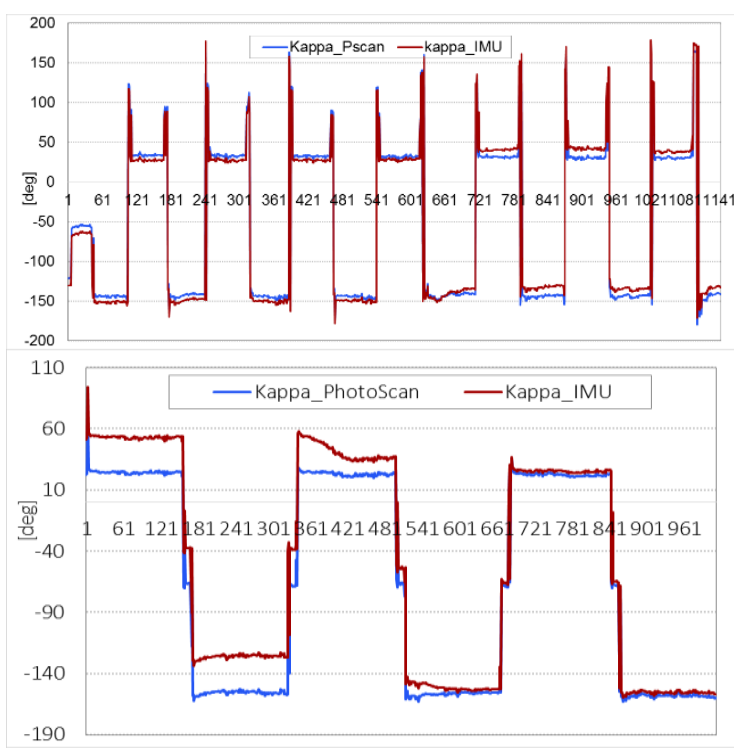

Figure 5. Camera orientation kappa angle estimated in real-time and in PhotoScan for Jokioinen (top) and Hyytiälä (bottom) area.

In order to assess the performance of our system, the real time georeferencing data from GNSS and IMU were used to generate spectral mosaics and NDVI index maps (Figure 9). These processes were performed as offline evaluation, but can be done as an online processing in drone. Using the GCPs to evaluate the quality of the orthomosaics, the RMSEs were $0.82 \mathrm{~m}$ in $\mathrm{E}$ and $1.2 \mathrm{~m}$ in $\mathrm{N}$, for Jokioinen test site and $1.9 \mathrm{~m}$ in $\mathrm{E}$ and $1.7 \mathrm{~m}$ in N, for Hyytiälä test site. As can be seen in Figure 6 (a), some parts of the field plantation lines are rotated as well as the road in forest (Figure $6 \mathrm{~d}$ ). The main cause of these problems is due to the non-accurate attitude estimation. Besides, the impact of the illumination condition can be seen in the real-time orthomosaic generation, and it is very clear in Jokioinen data set (Figure $6 \mathrm{c}$ ). This was corrected in the post-processing phase using radiometric block adjustment, as showed in Figure 6 (c) and (f). However, visual analysis of real-time drone based orthomosaics and index maps can already provide valuable information for expert users, such as farmers and foresters. Small anomalies in vegetation, caused for instance by drought, 
disease outbreak or pest invasion, can be detected, due to high spatial and spectral resolution of data.
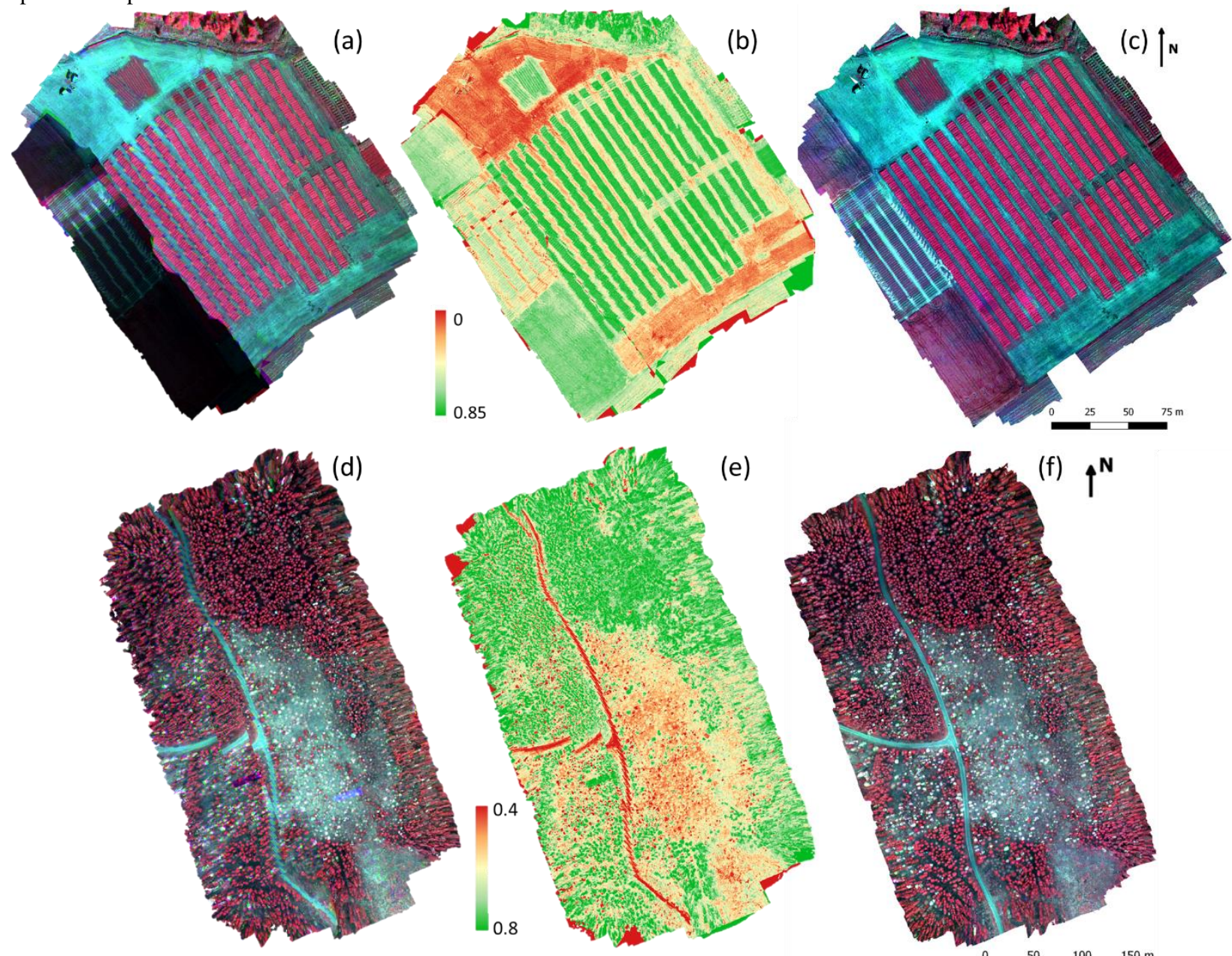

(e)

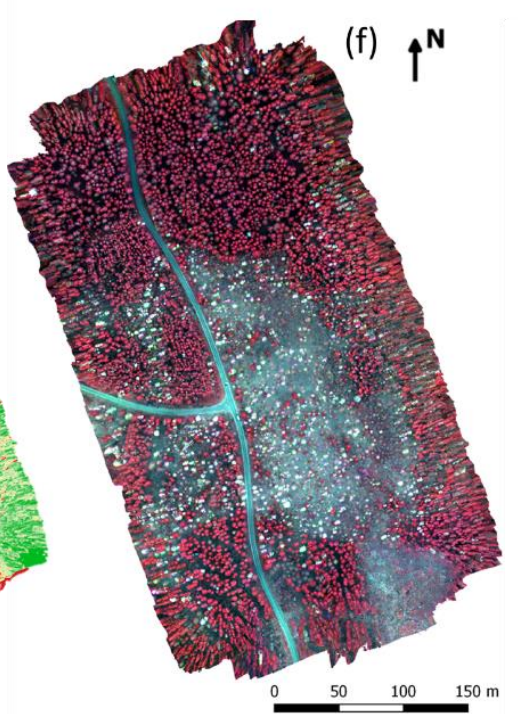

Figure 6. Orthomosaics of Jokioinen test site (a) Real-time CIR mosaic, (b) NDVI real-time mosaic and (c) post processed CIR mosaic; and orthomosaics of Hyytiälä test site (d) Real-time CIR mosaic, (e) NDVI real-time mosaic and (f) post processed CIR mosaic.

\subsection{Incremental BBA}

Real-time structure estimation and bundle block adjustment is performed on Vihti dataset for both local paths and the complete area. Figure 7 demonstrate a local approximation of few images on a turn. In this figure, the sparse point-cloud is painted in red. The error ellipses of a minimum-constraint block bundle adjustment of a local path of few images in demonstrated in Figure 8. The result of the complete sparse block bundle adjustment of 245 images is demonstrated in Figure 9.

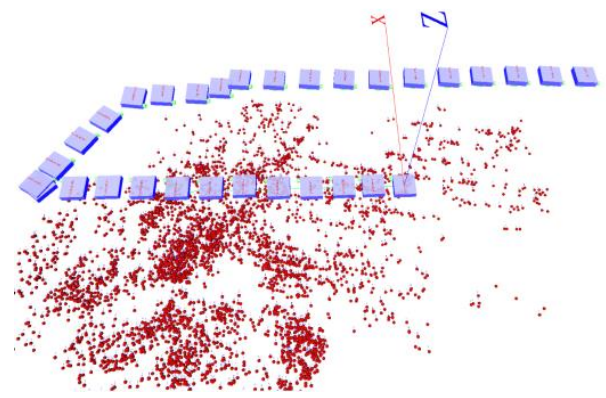

Figure 7. A local bundle block adjustment on Vihti dataset.

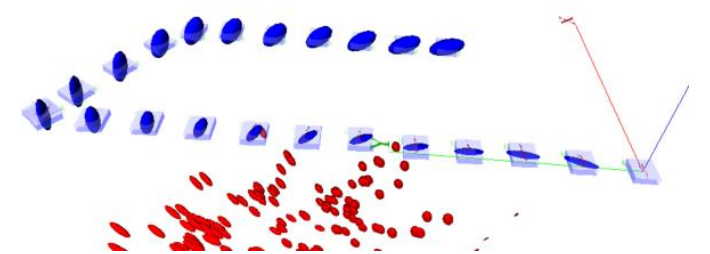

Figure 8. 3D Error ellipses of a minimum-constraint block bundle adjustment of few images in Vihti dataset 


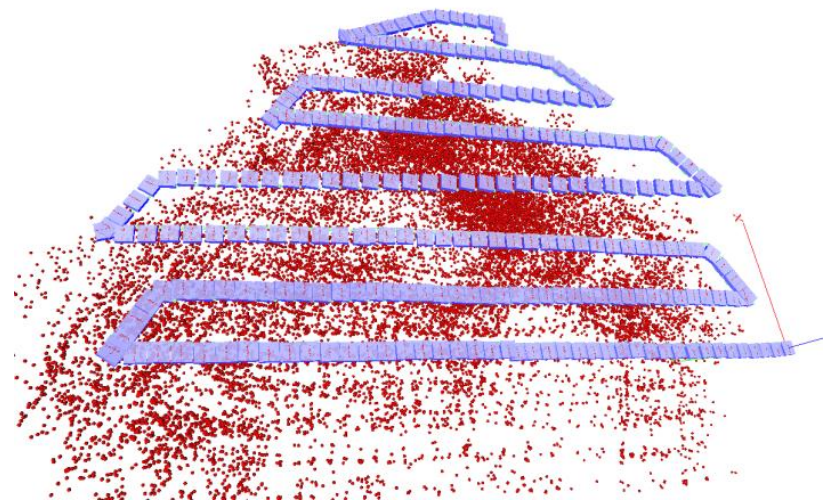

Figure 9. Sparse bundle block adjustment of 245-images of Vihti dataset.

\section{CONCLUSIONS}

A real-time direct georeferencing system using low cost GNSS/IMU sensors was established and the geometric performance was assessed in two different remote sensing scenarios. We have demonstrated that the real-time drone processing is feasible and an advantageous approach for rapidly assessing the status of an agriculture field and forest area. The implemented incremental BBA was shown to be promising to increase the real-time georeferencing solution provide by GNSS/IMU sensors and to be used in GNSS denied environments. We will implement more accurate GNSS/IMU system to obtain more accurate position and attitude solutions. We will improve the real-time bundle block adjustment solution and integrate all into a real-time processing chain. A novel realtime capable hyperspectral frame camera will be employed in the future works, since the hyperspectral camera used in this paper does not allow real-time accessing to the images. Furthermore, an irradiance sensor has been implemented to the real-time solution in order to correct influence of varying illumination to reflectance.

\section{ACKNOWLEDGEMENTS}

The authors would like to acknowledge Tekes - the Finnish Funding Agency for Innovation (DroneKnowledge-project; Dnro 1617/31/2016)).

\section{REFERENCES}

Cadena, C.; Carlone, L.; Carrillo, H.; Latif, Y.; Scaramuzza, D.; Neira, J.; Reid, I. and Leonard, J.J.; 2016. Past, present, and future of simultaneous localization and mapping: Toward the robust-perception age. IEEE Transactions on Robotics, 32, pp.1309-1332. https://doi.org/10.1109/TRO.2016.2624754

Cubert, 2018. http://cubert-gmbh.com/productcategory/spectral-cameras/. (16 March 2018).

Eling, C., Klingbeil, L. and Kuhlmann, H., 2015. Real-time single-frequency GPS/MEMS-IMU attitude determination of lightweight UAVs. Sensors, 15(10), pp. 26212-26235.

Forster, C., Carlone, L., Dellaert, F. and Scaramuzza, D., 2017. On-Manifold Preintegration for Real-Time Visual-Inertial Odometry. IEEE Transactions on Robotics, 33(1), pp.1-21.

Gruen, A., 1988. Towards real-time photogrammetry. Photogrammetria, 42(5-6), pp.209-244.
Honkavaara, E.; Saari, H.; Kaivosoja, J.; Pölönen, I.; Hakala, T.; Litkey, P.; Mäkynen, J.; Pesonen, L., 2013. Processing and Assessment of Spectrometric, Stereoscopic Imagery Collected Using a Lightweight UAV Spectral Camera for Precision Agriculture. Remote Sensing, 5(10), pp. 5006-5039.

Honkavaara, E. Khoramshahi, E., 2018. Radiometric Correction of Close-Range Spectral Image Blocks Captured Using an Unmanned Aerial Vehicle with a Radiometric Block Adjustment. Remote Sensing, 10(2), p.256. https://doi.org/10.3390/rs10020256

Honkavaara, E., Rosnell, T., Oliveira, R., Tommaselli, A., 2017. Band registration of tuneable frame format hyperspectral UAV imagers in complex scenes. ISPRS Journal of Photogrammetry and Remote Sensing, 134, pp.96-109. https://doi.org/10.1016/j.isprsjprs.2017.10.014

Kaess, M., Johannsson, H., Roberts, R., Ila, V., Leonard, J., Dellaert, F., 2012. iSAM2: Incremental Smoothing and Mapping Using the Bayes Tree. Intl. J. of Robotics Research (IJRR), Vol. 31, pp. 217-236.

Kang, S. and Lee, K., 2016. Auto-scaling of Geo-based image processing in an OpenStack cloud computing environment Remote Sensing, 8(8), p.662. https://doi.org/10.3390/rs8080662

Khoramshahi, Ehsan and Honkavaara, Eija, 2018. Modelling and Automated Calibration of a General Multi-Projective Camera. Photogrammetric Record. https://doi.org/10.1111/phor.12230.

Khoramshahi, E., Honkavaara, E. and Rosnell, T., 2017. An Automatic Method for Adjustment of a Camera Calibration Room. International Federation of Surveyors Working Week, Helsinki, Finland. 9 pages.

Kirkko-Jaakkola, M., Saarimäki, J., Söderholm, S., Guinness, R., Ruotsalainen, L., Kuusniemi, H., Koivula, H., Mattila, T., Nyberg, S., 2014. P3: A public precise positioning service based on a national GNSS network. International Conference on Localization and GNSS, Helsinki, Finland. pp. 1-5.

Koirala, P., Løke, T., Baarstad, I., Fridman, A. and Hernandez, J., 2017, May. Real-time hyperspectral image processing for UAV applications, using HySpex Mjolnir-1024. In Algorithms and Technologies for Multispectral, Hyperspectral, and Ultraspectral Imagery XXIII International Society for Optics and Photonics. Vol. 10198, pp. 1019807. https://doi.org/10.1117/12.2267476

Luo, C., Nightingale, J., Asemota, E. and Grecos, C., 2015. A UAV-cloud system for disaster sensing applications. Proceedings of the IEEE Vehicular Technology Conference (VTC Spring), Glasgow, Scotland, pp. 1-5.

Meier, L., Tanskanen, P., Fraundorfer, F. and Pollefeys, M., 2011, May. Pixhawk: A system for autonomous flight using onboard computer vision. Proceedings of the IEEE international conference Proceedings of the Robotics and automation (ICRA), Shanghai, pp. 2992-2997.

Oliveira, R., Tommaselli, A., Honkavaara, E., 2016. Geometric Calibration of a Hyperspectral Frame Camera. Photogrammetric Record. 31(155), pp. 325-347. https://doi.org/10.1111/phor.12153. 
Rizos, C. and Han, S., 2003. Reference station network based RTK systems-concepts and progress. Wuhan University Journal of Natural Sciences, 8(2), pp. 566-574.

Schneider, J., Eling, C., Klingbeil, L., Kuhlmann, H., Förstner, W. and Stachniss, C., 2016, May. Fast and effective online pose estimation and mapping for UAVs. Proc. of the IEEE International Conference on Robotics and Automation (ICRA), Stockholm, Sweden, pp. 4784-4791. https://doi.org/10.1109/ICRA.2016.7487682.

Senop, 2018. http://senop.fi/en/optronics-hyperspectral. (16 March 2018)

Sensefly, 2018. https://www.sensefly.com/drones/ (16 March 2018)

Skycatch, 2018. https://www.skycatch.com/precision-package/ (16 March 2018)

Suomalainen, J., Anders, N., Iqbal, S., Roerink, G., Franke, J., Wenting, P., Hünniger, D., Bartholomeus, H., Becker, R. and Kooistra, L., 2014. A lightweight hyperspectral mapping system and photogrammetric processing chain for unmanned aerial vehicles. Remote Sensing, 6(11), pp. 11013-11030.

Wu C. A GPU implementation of David Lowe's Scale Invariant Feature Transform. https://github.com/pitzer/SiftGPU (16 March 2018). 